\title{
Behavioral primary prevention of cardiovascular diseases
}

\author{
Andrew Elagizi ${ }^{1}$, Edward Archer ${ }^{2}$, Carl J. Lavie ${ }^{3}$ \\ ${ }^{1}$ Department of Internal Medicine, Leonard J Chabert Medical Center, Houma, LA, USA; ${ }^{2}$ EnduringFX, Columbia, SC, USA; ${ }^{3}$ Department of \\ Cardiovascular Diseases, John Ochsner Heart and Vascular Institute, Ochsner Clinical School-The University of Queensland School of Medicine, \\ New Orleans, LA, USA \\ Correspondence to: Carl J. Lavie, MD, FACC, FACP, FCCP, FESPM. Department of Cardiovascular Diseases, John Ochsner Heart and Vascular \\ Institute, Ochsner Clinical School-The University of Queensland School of Medicine, 1514 Jefferson Highway, New Orleans, LA 70121-2483, USA. \\ Email: clavie@ochsner.org. \\ Provenance: This is an invited Editorial commissioned by Editor-in-Chief Yilei Mao (Department of Liver Surgery, Peking Union Medical College \\ Hospital, Chinese Academy of Medical Sciences, Beijing, China). \\ Comment on: Patnode CD, Evans CV, Senger CA, et al. Behavioral Counseling to Promote a Healthful Diet and Physical Activity for Cardiovascular \\ Disease Prevention in Adults Without Known Cardiovascular Disease Risk Factors: Updated Evidence Report and Systematic Review for the US \\ Preventive Services Task Force. JAMA 2017;318:175-93.
}

Submitted Oct 19, 2017. Accepted for publication Nov 28, 2017.

doi: 10.21037/hbsn.2017.12.02

View this article at: http://dx.doi.org/10.21037/hbsn.2017.12.02

The 2012 grade $\mathrm{C}$ recommendation from the United States Preventive Service Task Force (USPSTF) is that clinicians may choose to selectively counsel adults about healthful diet and physical activity (PA) for cardiovascular disease (CVD) prevention, in adults without known CVD, hypertension (HTN), diabetes mellitus (DM) or dyslipidemia (1). A recent review was published in $7 A M A$ to update the USPSTF behavioral counseling recommendation (2). The review by Patnode $e t$ al. (2) analyzed data from 88 randomized clinical trials $(\mathrm{n}=121,190)$ studying behavioral interventions targeting diet, PA, sedentary time or a combination thereof, finding no consistent benefit in all-cause or CVD morbidity or mortality. There were small, statistically significant differences in risk factors such as blood pressure (BP), body mass index (BMI), low-density lipoprotein (LDL) and total cholesterol levels at 6-12 months, with a dose-response effect to intervention, however, very limited evidence on longer-term health outcomes. We argue that there may still be insufficient evidence to guide caregivers to routinely counsel patients with behavioral interventions, at least regarding dietary recommendations, for primary prevention for CVD, whereas the PA evidence appears stronger.

It has been estimated that $>50 \%$ global deaths can be attributed to diet, and that if the US population consumed an average $1,500 \mathrm{mg} /$ day of sodium, there would be a $25.6 \%$ overall decrease in high BP and $\$ 26.2$ billion in healthcare savings (3). While the study of dietary effects on health is important, we previously demonstrated the often fatal flaws in nutrition studies which were used in the formulation of national dietary guidelines $(4,5)$. The most important finding in the review by Patnode et al. (2) was regarding the four trials that focused on "high-intensity diet interventions", and found no differences in all-cause or CVD-related mortality over 3-15 years follow-up. These results suggest that either high-intensity dietary change interventions are ineffective at effecting actual behavior change, or, as we previously posited, diet is a trivial risk factor for mortality $(4,6)$, and therefore, actual changes in dietary behavior will have no discernable impact on actual CVD mortality.

There are important limitations of the initial report, considering that "almost all of the behavioral outcomes were based on self-report" and "dietary intake was most often measured by food frequency questionnaires... or 24-hour food recalls". We previously argued that these memory-based assessment methods (M-BMs) are both pseudoscientific and inadmissible as scientific evidence (4-7). Because M-BM data are often physiologically implausible and "incompatible with survival" (7), they should not be used to quantify the effectiveness of interventions. Further, the lack of credibility of M-BM data has been replicated consistently over the past 3 decades (5). Thus, the pooling of results between the 
self-reported outcomes and the limited number of studies that used objective measures of behavior change (e.g., pedometers, accelerometers) obscured potentially valid results. Stated simply, the use of self-report precludes the research and medical community's ability to gain insight into the actual effect of behavioral counseling on all-cause and CVD mortality.

The conclusion, while not wholly inaccurate, obscured the finding that high-intensity diet interventions do not appear to provide any benefit on all-cause and CVD mortality. Presenting conclusions based on proxy outcomes rather than focusing on actual differences in mortality is potentially misleading because it is well established that many proxies (e.g., total cholesterol, adiposity) have little or no predictive value with respect to actual mortality (8). In the conclusion of the report, the authors stated there were "consistent modest benefits across a variety of important intermediate bealth outcomes across 6 to 12 months..." despite the fact that no benefits in actual mortality over much longer periods were demonstrated, which is disingenuous.

\section{Evidence for behavioral intervention as primary prevention of CVD}

Obesity and DM show disturbing trends of not only increasing but also affecting people at an earlier age (3). Declines in PA and increased sedentary behaviors have led to poor physical and mental health at progressively younger ages such that children and adolescents present with metabolic and $\mathrm{CV}$ problems previously limited to adults, such as DM, atherosclerosis, HTN, hyperlipidemia and impaired fasting glucose (9). Preclinical substrates for clinical CVD (e.g., fatty streaks and atherosclerosis) begin early in life and are influenced over time by modifiable risk factors, behaviors and environmental exposures (3). The high direct medical care and indirect costs of CVD, approaching \$450 billion per year in 2010 and projected to rise to over $\$ 1$ trillion per year by 2030 makes primary and primordial prevention of CVD a critical medical and societal issue (3). Most of this cost is attributable to shortand long-term care, not prevention.

It was reported that low PA is responsible for $6 \%$ of deaths globally (the fourth leading risk factor for mortality) and has been described as one of the greatest public health challenges of our time (9). Numerous studies demonstrate the association between low PA and higher CVD risk factors, such as HTN, obesity, hyperlipidemia and DM, as well as coronary heart disease (CHD), mortality and various cancers (10). It would seem that one is hard pressed to find any disease today that is not helped in some fashion by engaging in regular PA (11).

The importance of cardiorespiratory fitness (CRF), which is a plateau in oxygen uptake, above which further increases in exercise intensity produce no additional increase in oxygen uptake, has often been neglected in the equation of major CHD and CVD risk, despite the fact that it appears to be one of the most important correlates of overall health status and a potent predictor of an individual's future risk of CVD (12). It has been shown that CRF largely negates the adverse effects of traditional CHD risk factors, and an important feature of the CRF-CVD relationship is that CRF does not need to be exceptionally high to provide significant protection (12). In most cases, patients with major CHD risk factors (obesity, DM, HTN, etc.) and high CRF have lower mortality than patients without these risk factors but low CRF (12). There is accumulating evidence that physically active people are at lower risk for all-cause mortality than physically inactive ones, regardless of BMI. Overweight/obese individuals who are active have as low and often lower risk of morbidity and mortality than normal-weight sedentary individuals (11).

PA is often neglected in clinical practice; PA is often not discussed, documented in the medical record or prescribed. Since 2010, several large health care institutions have included PA as a vital sign (e.g., Kaiser Permanente) (11). The importance of CRF has been noted such that a policy statement from the AHA suggested the need for a CRF database (12). Unfortunately, despite the demonstrated importance of CRF, exercise testing is currently not reimbursed for routine use in individuals lacking certain medical conditions.

Small improvements in CRF often translate into substantial benefits regarding overall health, morbidity and mortality. For example, each 1 metabolic equivalent (MET) increase has been associated with a large (10-25\%) improvement in survival (12). Interestingly, the largest impact and health benefits were observed in the least fit groups of multiple studies.

Previous guidelines called for a minimum of 150 minutes per week of moderate-intensity aerobic PA or 75 minutes per week of vigorous-intensity PA, however, more recent evidence suggests substantial benefits are obtained with exercise training doses much lower than these guidelines (10). The use of pedometers (e.g., Fitbit) to track PA is becoming increasingly widespread; 10,000 steps per day is often cited without substantial evidence, however, a recent review 
concluded that $7,000-8,000$ steps/day meets minimal PA guidelines (13). It is important to understand that current recommendations for PA are a target, not a threshold (11). We recently argued that PA that increases heart rate is particularly helpful, and using a Personalized Activity Intelligence (PAI) and obtaining at least 50 points per week and preferably 100 points can provide maximal protection against CVD- and all-cause mortality $(14,15)$.

There is mounting evidence that PA in doses far lower than current recommendations is also beneficial, and that patients with the lowest level of CRF experience the greatest survival benefit by improving their CRF. A study from the ACLS (16) followed 55,000 people (13,000 runners and 42,000 non-runners) over a mean of 15 years, and found that runners had $30 \%$ and $45 \%$ decreased all-cause and CVD mortality, respectively. Interestingly, the quintile with the lowest doses of training ( $<6$ miles per week, 1-2 times per week, $<51$ minutes per week) had similar reductions to quintiles $2-4$ and slightly greater benefit than quintile 5 . These results suggest that with running, the maximal effect on all-cause and CVD mortality occurs at low doses, well below the current major PA guidelines (16-19). Further analysis from the ACLS study has also shown an inverse correlation between CRF and all-cause mortality; the largest reduction in all-cause mortality occurred between the lowest 2 CRF quintiles, again suggesting that the least fit cohort could receive the greatest survival benefit by increasing their PA (and therefore, increasing CRF) (20). Multiple studies also demonstrate the importance of higher CRF at younger age, which seems to confer the greatest survival benefit (20). Although there are variations by race and sex in CRF, the potential benefit of CRF on mortality is independent of these factors (20). CRF may be the best single measure of overall health status, as its measurement involves multiple physiological conditions and is affected by various disease states (20).

\section{Conclusions}

The studies reviewed by Patnode et al. (2) regarding behavioral counseling for diet, PA and sedentary lifestyle for CVD prevention in adults without known CVD or CVD risk factors concluded (I) no consistent benefit in allcause or CVD morbidity or mortality; (II) small, statistically significant differences in intermediate outcomes; and (III) very limited evidence on longer-term intermediate and health outcomes. Considering that most studies that evaluate dietary patterns rely on M-BMs, which have been shown to be pseudoscientific and inadmissible as scientific evidence (4-7), there continues to be a void in the field of nutrition research for scientifically sound data to implement federal regulations and national guidelines. The conclusions from the review regarding high-intensity diet interventions would suggest that high-intensity dietary change interventions are either ineffective at effecting actual behavior change, or that diet is a trivial risk factor for mortality. Further study is required with more scientific means of collecting data, in order to obtain clear evidence regarding the association between diet and CVD as well as overall health, and for the implementation of scientifically sound national guidelines regarding healthful diet.

CVD continues to be a leading cause of death and disability, and there is evidence that CVD is largely preventable. The direct and indirect medical costs of CVD continue to increase, making primary prevention a critical medical and societal issue. As most of the cost is attributable to short- and long-term care, rather than prevention, exercise should be viewed as a safe, cost-effective medication that is universally prescribed as a first-line treatment for virtually every chronic disease (11). Physical inactivity has been said to be the fourth leading risk factor for mortality, and is associated with various disease processes, including CVD and various cancers $(9,12)$; certainly, PA is extremely cost effective, and lack of PA is extremely costly.

Low CRF is a poorly appreciated but exceedingly important risk factor that is modifiable without reliance on further diagnostic or costly therapeutic interventions (12). It was shown that CRF largely negates the adverse effects of traditional CVD risk factors, such that individuals with major risk factors who are physically active (higher CRF) typically have equal or lower morbidity and mortality than their sedentary counterparts who lack risk factors. While many physicians neglect PA altogether, the evidence for CRF is so compelling that the AHA has advocated for the use of CRF as a vital sign, and for establishment of a CRF database.

Perhaps most importantly regarding PA and CRF, there appears to be substantial benefits with minor improvements, particularly in those who have the lowest levels of CRF. Further, benefits from PA are shown at levels far below current guidelines, and current PA recommendations should be regarded as a target, not a threshold. These data are very encouraging and suggest major improvements in population level morbidity, mortality and health care costs by implementing Exercise as Medicine. 


\section{Acknowledgements}

None.

\section{Footnote}

Conflicts of Interest: The authors have no conflicts of interest to declare.

\section{References}

1. Final Recommendation Statement: Healthful Diet and Physical Activity for Cardiovascular Disease Prevention in Adults: Behavioral Counseling. Available online: https:// www.uspreventiveservicestaskforce.org/Page/Document/ RecommendationStatementFinal/healthful-diet-andphysical-activity-for-cardiovascular-disease-prevention-inadults-behavioral-counseling

2. Patnode CD, Evans CV, Senger CA, et al. Behavioral Counseling to Promote a Healthful Diet and Physical Activity for Cardiovascular Disease Prevention in Adults Without Known Cardiovascular Disease Risk Factors: Updated Evidence Report and Systematic Review for the US Preventive Services Task Force. JAMA 2017;318:175-93.

3. Weintraub WS, Daniels SR, Burke LE, et al. Value of primordial and primary prevention for cardiovascular disease: a policy statement from the American Heart Association. Circulation 2011;124:967-90.

4. Archer E, Pavela G, Lavie CJ. The Inadmissibility of What We Eat in America and NHANES Dietary Data in Nutrition and Obesity Research and the Scientific Formulation of National Dietary Guidelines. Mayo Clin Proc 2015;90:911-26.

5. Archer E, Pavela G, Lavie CJ. A Discussion of the Refutation of Memory-Based Dietary Assessment Methods (M-BMs): The Rhetorical Defense of Pseudoscientific and Inadmissible Evidence. Mayo Clin Proc 2015;90:1736-9; discussion 1739-40.

6. Archer E, Marlow ML, Williams RA. Government Dietary Guidelines: Uncertain Science Leads to Questionable Public Health Policy. Available online: https://www. mercatus.org/system/files/mercatus-archer-gov27tdietary-guidelines-v1.pdf

7. Archer E, Hand GA, Blair SN. Validity of U.S. nutritional surveillance:National Health and Nutrition Examination Survey caloric energy intake data, 1971-2010. PLoS One 2013;8:e76632.

8. Lavie CJ, De Schutter A, Parto P, et al. Obesity and Prevalence of Cardiovascular Diseases and Prognosis-
The Obesity Paradox Updated. Prog Cardiovasc Dis 2016;58:537-47.

9. Hills AP, Dengel DR, Lubans DR. Supporting public health priorities: recommendations for physical education and physical activity promotion in schools. Prog Cardiovasc Dis 2015;57:368-74.

10. Lavie CJ, Arena R, Swift DL, et al. Exercise and the cardiovascular system: clinical science and cardiovascular outcomes. Circ Res 2015;117:207-19.

11. Sallis R, Franklin B, Joy L, et al. Strategies for promoting physical activity in clinical practice. Prog Cardiovasc Dis 2015;57:375-86.

12. Kaminsky LA, Arena R, Beckie TM, et al. The importance of cardiorespiratory fitness in the United States: the need for a national registry: a policy statement from the American Heart Association. Circulation 2013;127:652-62.

13. Tudor-Locke C, Leonardi C, Johnson WD, et al. Accelerometer steps/day translation of moderate-tovigorous activity. Prev Med 2011;53:31-3.

14. Nes BM, Gutvik CR, Lavie CJ, et al. Personalized Activity Intelligence (PAI) for Prevention of Cardiovascular Disease and Promotion of Physical Activity. Am J Med 2017;130:328-36.

15. Zisko N, Skjerve KN, Tari AR, et al. Personal Activity Intelligence (PAI), Sedentary Behavior and Cardiovascular Risk Factor Clustering - the HUNT Study. Prog Cardiovasc Dis 2017;60:89-95.

16. Lee DC, Pate RR, Lavie CJ, et al. Leisure-time running reduces all-cause and cardiovascular mortality risk. J Am Coll Cardiol 2014;64:472-81.

17. Lavie CJ, Lee DC, Sui X, et al. Effects of Running on Chronic Diseases and Cardiovascular and All-Cause Mortality. Mayo Clin Proc 2015;90:1541-52.

18. Lee DC, Lavie CJ, Sui X, et al. Running and Mortality: Is More Actually Worse? Mayo Clin Proc 2016;91:534-6.

19. Lee DC, Brellenthin AG, Thompson PD, et al. Running as a Key Lifestyle Medicine for Longevity. Prog Cardiovasc Dis 2017;60:45-55.

20. Harber MP, Kaminsky LA, Arena R, et al. Impact of Cardiorespiratory Fitness on All-Cause and DiseaseSpecific Mortality: Advances Since 2009. Prog Cardiovasc Dis 2017;60:11-20.

Cite this article as: Elagizi A, Archer E, Lavie CJ. Behavioral primary prevention of cardiovascular diseases. HepatoBiliary Surg Nutr 2018;7(1):34-37. doi: 10.21037/hbsn.2017.12.02 